\title{
POINTS NOT AS HYPERPLANE SECTIONS OF PROJECTIVELY NORMAL CURVES
}

\author{
EDOARDO BALLICO
}

(Communicated by Louis J. Ratliff, Jr.)

\begin{abstract}
Here we show for many $n, d$ (with $n \geq 6$ ) that the general set formed by $d$ points in $\mathbf{P}^{n}$ is not the hyperplane section of an integral projectively normal curve in $\mathbf{P}^{n+1}$.
\end{abstract}

Here we give a negative answer to a part of a question raised in [EEK, p.53] proving the following theorem:

Theorem A. Fix an integer $n \geq 6$. Then there is an integer $d$ such that, for a general set $S$ of $d$ points in $\mathbf{P}^{n}$, there is no arithmetically Cohen-Macaulay integral locally complete intersection curve $C \subset \mathbf{P}^{n}$ with $S$ as one of its hyperplane sections; more precisely, this occurs for all $n, d$ satisfying the following two inequalities:

$$
\begin{gathered}
d \leq(n+2)(n+1) / 2, \\
(n-4) d>(n-2)(n+2)
\end{gathered}
$$

and for all $n, d$ such that there is an integer $m$ with $(n, d, m)$ satisfying the following conditions:

$$
m \geq 3, \quad n \geq 4 m+4, \quad \text { and }\left(\frac{n+m-1}{n}\right)<d \leq\left(\frac{n+m}{n}\right) .
$$

Note that an affirmative answer to a related question (without the assumption "Cohen-Macaulay" in the statement of Theorem A) was given in [BM]. The proof of Theorem A will be just a dimensional count; however, the statement itself is interesting, and several mathematicians have asked about it. It is known to be false if $n=2[\mathrm{CO}]$.

Proof of Theorem A. Fix integers $n, d$ satisfying (1) and (2) with $n \geq 2$ (hence $n \geq 6$ ), and consider $\mathbf{P}^{n}$ as a hyperplane $H$ of $\mathbf{P}=\mathbf{P}^{n+1}$. The set of $d$ points in $H$ has dimension $n d$. Fix any set $S$ of $d$ points imposing for

Received by the editors January 31, 1990 and, in revised form, May 16, 1990.

1980 Mathematics Subject Classification (1985 Revision). Primary 14H99, 14N05.

Key words and phrases. Hyperplane section, projective normal curve, arithmetically CohenMacaulay curve, normal sheaf. 
all $t \geq 2$ independent conditions on the forms of degree $t$ in $H$ (i.e., such that, in standard notation, $h^{1}\left(H, \mathbf{I}_{S, H}(t)\right)=0$ for every $\left.t \geq 2\right)$; in particular, we may take as $S$ a general set of $d$ points of $H$. Assume the existence of an arithmetically Cohen-Macaulay integral curve $C \subset \mathbf{P}^{n+1}, \operatorname{deg}(C)=d$, with $C \cap H=S$; let $\mathbf{I}_{C}$ be the ideal sheaf of $C$ in $\mathbf{P}^{n+1}$. From the exact sequences

$$
0 \rightarrow \mathbf{I}_{C}(t-1) \rightarrow \mathbf{I}_{C}(t) \rightarrow \mathbf{I}_{S, H}(t) \rightarrow 0
$$

and descending induction on $t$, we get $h^{2}\left(\mathbf{P}^{n+1}, \mathbf{I}_{C}(1)\right)=0$; i.e., $h^{1}\left(C, \mathbf{O}_{C}(1)\right.$ $=0$. Thus, since $C$ is linearly normal, its arithmetic genus $g$ is $d-n-1$. Let $N_{C}$ be the normal sheaf $\left(\mathbf{I}_{C} / \mathbf{I}_{C}^{2}\right)^{*}$ to $C$ in $\mathbf{P}^{n+1}$. By the Euler's sequence of $T \mathbf{P}^{n+1}$, there is a map t: $(n+2) \mathbf{O}_{C}^{n}(1) \rightarrow N_{C}$ with $\operatorname{Coker}(\mathbf{t})$ supported at the singular points of $C$, hence with $\operatorname{dim}(\operatorname{Supp}(\operatorname{Coker}(\mathbf{t})))=0$. Thus $h^{1}\left(N_{C}\right)=$ 0 . Hence $h^{0}\left(N_{C}\right)=\chi\left(N_{C}\right)$. Since $H^{0}\left(C, N_{C}\right)$ is the tangent space to the Hilbert scheme $\operatorname{Hilb}\left(\mathbf{P}^{n+1}\right)$ at the point $C, h^{0}\left(N_{C}\right)$ is an upper bound for the irreducible components of $\operatorname{Hilb}\left(\mathbf{P}^{n+1}\right)$ containing $C$. If $C$ is a locally complete intersection, by [H, Chapter III, Theorem 7.1], we have $\omega_{C}=\omega_{\mathbf{P}} \otimes c_{1}\left(N_{C}\right)$. Thus $\chi\left(N_{C}\right)=(n+2) d+(n-2)(1-g)$. Putting $g=d-n-1$, we see that the set of intersections $\{T \cap H\}$ with $T$ in an irreducible component of Hilb $\left(\mathbf{P}^{n+1}\right)$ containing $C$ cannot contain an open dense set of the symmetric product $S^{d}(H)$ if (2) holds.

Now fix integers $n, m$, and $d$ satisfying (3). Fix $S \subset H$ with $\operatorname{card}(S)=d$, and an integral locally complete intersection arithmetically CohenMacaulay curve $C \subset \mathbf{P}^{n+1}$ with $\operatorname{deg}(C)=d$ and $S=C \cap H$; now we assume $h^{1}\left(H, \mathbf{I}_{S, H}(z)\right)=0$ for $z \geq m$ and $h^{0}\left(H, \mathbf{I}_{S, H}(t)\right)=0$ if $t<m$; thus $h^{1}\left(H, \mathbf{I}_{S, H}(w)\right)=d-h^{0}\left(H, \mathbf{O}_{H}(w)\right)$ if $0 \leq w<m$. Of course, by (3) these assumptions are satisfied if $S$ is a general set of $d$ points in $H$. Set $h^{1}=h^{1}\left(\mathbf{O}_{C}(1)\right)$. Now we have $g:=p_{a}(C)=d+h^{1}-1-n$ by Riemann-Roch. By the classical proof of Castelnuovo's bound for the genus [H2, Chapter 3] and the fact that $C$ is arithmetically Cohen-Macaulay, we have

$$
h^{1}=\sum_{t=2}^{m-1} h^{1}\left(H, \mathbf{I}_{S, H}(t)\right) \text {. }
$$

Using the very rough bound $h^{1}\left(N_{C}\right) \leq(n+2) h^{1}$, which comes from the Euler's sequence and the finiteness of $\operatorname{Supp}(\operatorname{Coker}(t))$, we get the last part of Theorem A.

Remark 1. In the proof of Theorem A, we used the assumption that the curve is a locally complete intersection only to compute $\chi\left(N_{C}\right)$. This computation depends only on a formal neighborhood of the singularities of $C$. Thus, by $[\mathrm{K}$, Lemma 2.2 .11$, p. 95], it is sufficient to assume that every nonlocally complete intersection point of $C$ has embedding dimension 3. Furthermore, if we fix a measure $\delta$ of the failure of this local computation at the nonlocally complete 
intersection points, we can find $n, d$ as in (1) for which the general $S$ is not a hyperplane section of an integral arithmetically Cohen-Macaulay curve with degree of failure of $\chi\left(N_{C}\right)$ at most $\delta$ if instead of (2) we assume $(2)^{\prime}$, which is as (2) except for a term $+\delta$ on the right-hand side.

We conjecture that for every $n \geq 6$ there is a $d(n)$ such that, if $d \geq d(n)$, a general set of $d$ points of $\mathbf{P}^{n}$ is not the hyperplane section of an integral locally complete intersection arithmetically Cohen-Macaulay curve in $\mathbf{P}^{n+1}$; furthermore, it should be possible to drop the assumption that the curve is a locally complete intersection.

We would like to use this occasion to point out that the fact that the monodromy group of the general hyperplane section $S$ of an integral curve is big ([H1] and [R] if $\operatorname{char}(\mathbf{K})>0$ ) implies not only that $S$ is in uniform position, but also that any two subsets of $S$ with the same cardinality have isomorphic minimal free resolutions (i.e., the same Betti numbers). We would like to say that $S$ is syzygetic uniform position if this happens. Since the minimal free resolution of any hyperplane section of an arithmetically Cohen-Macaulay curve has as Betti numbers the Betti numbers of a minimal free resolution of the curve, this happens also in any characteristic for every hyperplane section of an integral arithmetically Cohen-Macaulay curve. Furthermore, if $k \leq n$ is an integer, we say that $S$ is an syzygetic uniform position up to step $k$ if there is an isomorphism between the first $k$ steps of the minimal free resolution of any two subsets with the same cardinality of $S$. Uniform position implies syzygetic uniform position for points in the plane by [CO], but not in general. The easiest example (the verification is left to reader) is for $n=3, d=8$; start with seven general points $P_{i}, 0 \leq i \leq 6$, in $\mathrm{P}^{3}$ (hence with homogeneous ideal generated by 3 quadrics and 1 cubic, e.g., by [B]); let $D$ be the rational smooth curve containing the points $P_{i}$ with $i>0$; take a general point $P_{7}$ on $D ;$ set $S=\left\{P_{i}\right\}_{0 \leq i \leq 7}$.

\section{ACKNOWLEDGMENTS}

I want to thank J. Migliore and G. Valla for several interesting conversations. This work was thought out and written while I was in the warm atmosphere of The Max-Planck Institute für Mathematik (Bonn): thanks!

\section{REFERENCES}

[B] E. Ballico, Generators for the homogeneous ideal of $s$ general points in $\mathbf{P}^{3}$, J. Algebra 106 (1987), 46-52.

[BM] E. Ballico and J. Migliore, Smooth curves whose hyperplane section is a given set of points, Comm. Algebra 18 (1990), 3015-3040.

[CO] L. Chiantini and F. Orecchia, Plane sections of arithmetically normal curves in $\mathbf{P}^{3}$, Algebraic Curves and Projective Geometry, Lecture Notes in Math., vol. 1389, Springer-Verlag, pp. 32-42.

[E] L. Ein, D. Eisenbud, and S. Katz, Varieties cut out by quadrics: Scheme theoretic versus homogeneous generation of ideals, Algebraic Geometry: Sundance 1986, Lecture Notes in Math., vol. 1311, Springer-Verlag, pp. 51-70. 
[H1] J. Harris, Galois groups of enumerative problems, Duke Math. J. 46 (1979), 685-724.

[H2] J. Harris with D. Eisenbud, Curves in a projective space, Les Presses de l'Universite de Montréal, 1982.

[HA] R. Hartshorne, Algebraic geometry, Graduate Texts in Math., vol. 52, Springer-Verlag, 1977.

[K] J. Kleppe, Deformations of graded algebras and of projective schemes of nests. Applications to the Hilbert scheme of curves in 3-space, Thesis, Oslo University, 1981.

[R] J. Rathmann, The uniform position principle for curves in characteristic $p$, Math. Ann. 276 (1987), 565-579.

Department of Mathematics, University of Trento, 38050 Povo (TN), Italy

E-mail address: ballico@itncisca.bitnet 